
Revue canadienne de chimie

\title{
Partitioning of Reactive Yellow 86 between Aqueous and Micellar Media studied by Differential Absorption Spectroscopy
}

\begin{tabular}{|r|l|}
\hline Journal: & Canadian Journal of Chemistry \\
\hline Manuscript ID & cjc-2016-0442.R1 \\
\hline Manuscript Type: & Article \\
\hline Complete List of Authors: & $\begin{array}{l}\text { HAQ, NAVEED; GC UNIVERSITY FAISALABAD, chemistry } \\
\text { USMAN, MUHAMMAD; GC UNIVRERSITY FAISALABAD, CHEMISTRY } \\
\text { HUSSAIN, AJAZ; Bahauddin Zakariya University } \\
\text { FAROOQI, ZAHOOR; University of the Punjab, chemistry } \\
\text { SAEED, MUHAMMAD ; GOVERNMENT COLLGE UNIVERSITY } \\
\text { HANIF, SADIA; GC UNIVERSITY, FAISALABAD, chemistry } \\
\text { IRFAN, MUHAMMAD; Government College University Faisalabad } \\
\text { Siddiq, Mohammad; Quaid-i-Azam University Islamabad, Chemistry } \\
\text { Rana, Usman Ali; King Saud University, Chemistry }\end{array}$ \\
\hline Please Select from this Special & \multicolumn{2}{|c|}{} \\
Issues list if applicable: & \multicolumn{2}{|c|}{ Keyword: } & dye, surfactant, solubilization, spectroscopy, micelle, partition coefficient. \\
\hline \multicolumn{2}{|c|}{} \\
\hline \multicolumn{2}{|c|}{} \\
\hline
\end{tabular}




\section{Partitioning of Reactive Yellow 86 between Aqueous and Micellar Media studied by}

\section{Differential Absorption Spectroscopy}

Naveed ul Haq ${ }^{a}$, Muhammad Usman $^{a,{ }^{*},}$ Ajaz Hussain ${ }^{b, c}$, Zahoor Hussain Farooqi ${ }^{d}$,

Muhammad Saeed ${ }^{a}$, Sadia Hanif ${ }^{a}$, Muhammad Irfan $^{a}$, Mohammad Siddiq $^{e}$, Usman Ali Rana ${ }^{f}$

${ }^{a}$ Department of Chemistry, Government College University, Faisalabad-38000, Pakistan.

${ }^{b}$ Department of Basic Sciences and Humanities, Khawaja Fareed University of Engineering and Information Technology, Rahim Yar Khan, Pakistan.

${ }^{c}$ Institute of Chemical Sciences, Bahauddin Zakariya University-60800, Multan, Pakistan. ${ }^{d}$ Institute of Chemistry, University of the Punjab, Lahore-54590, Pakistan.

${ }^{e}$ Department of Chemistry, Quaid-i-Azam University, Islamabad, Pakistan.

${ }^{f}$ Sustainable Energy Technologies Center, College of Engineering, King Saud University, PO-Box 800, Riyadh 11421, Kingdom of Saudi Arabia.

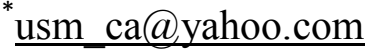

$+923017853113$ 


\section{Abstract}

23 aqueous and micellar media of a cationic surfactant, Cetyltrimethyl ammonium bromide (CTAB)

24 as well as an anionic surfactant, Sodium Dodecyl Sulphate, SDS. In a systematic investigation, 25 we have recorded the UV/Visible absorption spectra of the dye as a function of surfactant's 26 concentration above and below the critical micelle concentration (CMC). Absorption spectra 27 display red shift in case of CTAB and hypochromic shift upon using SDS. The partition 28 coefficient $\left(K_{x}\right)$ was calculated using differential absorption data and the value of free energy of 29 partition $\left(\Delta G_{p}\right)$ was calculated using this $K_{x}$ value. The results revealed that the dye is solubilized 30 in CTAB micelles to greater extent than in SDS micelles.

31 Key Words: Dye, surfactant, solubilization, spectroscopy, micelle, partition coefficient. 


\section{INTRODUCTION}

34 In recent years, the surfactants oriented research has undergone a dramatic development where

35 more emphasis has been focused to exploit the applications of surfactant systems to achieve

36 specific targets in chemical and technological areas of organic chemistry, physical chemistry,

37 petroleum and mineral processing, cosmetics and pharmaceuticals. Surfactants offer key role as

38 industrial cleaners, wetting, dispersing and leveling agents ${ }^{[1,2]}$ as well as they are potentially

39 useful for green chemistry applications, being used as micellar catalysts, where they have

40 replaced harmful organic solvents. ${ }^{[3-7]}$ One of the most common industrial applications of

41 surfactants can be found in textile industry as surfactants are widely used to improve dyeing

42 process by increasing solubility, stabilizing the dispersed state and promoting uniform

43 distribution of the dye. The use of surfactants is not only limited to adsorption and fixation of

44 dyes on substrate, but also to address environmental problems for the removal of loosely bound

45 dyes and other toxic organics from the substrate via solubilization process. ${ }^{[8,9]}$ The importance of

46 solubilization lies in the fact that it enables us to get rid of organic co-solvents that may may

47 contaminate the environment by causing pollution and may lead to health problems. ${ }^{[10,11]}$. The

48 intrinsic nature of micelles, being heterogeneous in nature for encapsulating polar as well as non-

49 polar compounds enables them to serve aforementioned purpose. ${ }^{[2,12]}$

50 In premicellar region, monomers of surfactant interact with the dye molecules to form ion

51 association complexes, while, in post micellar region dye molecules are likely to be incorporated

52 into micelles. ${ }^{[9]}$ Hydrophobic effect is dominant to decide the locus of additive within micelle,

53 although, the effects of electrostatic interactions are not too weak to be ignored. ${ }^{[13,14]}$ 
54 In 1954, Rattee and Stephens were the first to produce reactive dyes at the Imperial Chemical

55 Industries, Manchester, which were later introduced commercially in 1956. The chromophore

56 groups of these dyes are capable to directly interact with the substrate. ${ }^{[15]}$

57 The spectra of many dyes are sensitive to polarity of solvent, the phenomenon being called 58 solvatochromism. Positive solvatochromism exhibits red shift due to the enhanced polarity of the 59 environment around the dye, while in case of negative solvatochromism, a blue shift is often 60 observed. In positive solvatochromism, the excited state is more polar than the ground state, 61 while in negative solvatochromism, the ground state is more polar. ${ }^{[16]}$

62 Earlier, we reported the solubilization of some drugs such as Chloroquine Diphosphate, 63 and Pefloxacin mesylate using the micellar solutions. ${ }^{[17-18]}$ In the present study, we attempted to 64 explore the solubilization process of the reactive yellow 86 (RY 86) dye with a cationic 65 surfactant [Cetyltrimethyl ammonium bromide (CTAB)] and an anionic surfactant [Sodium 66 Dodecyl Sulphate (SDS)] using spectroscopic techniques. We anticipate that the observations 67 from this study will be helpful to choose the best optimized conditions for spontaneous 68 solubilization of dye in the micellar solution. Figure 1 represents the structure of the reactive 69 yellow 86. 


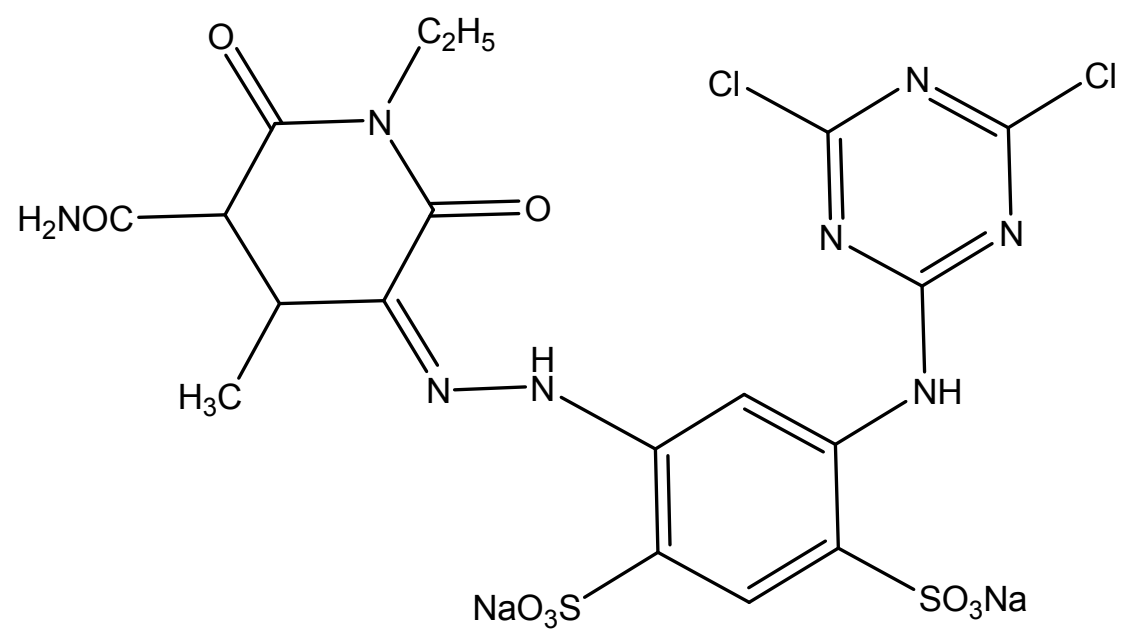

72 Figure1. Chemical structure of Reactive yellow 86

\section{MATERIALS AND METHODS}

74 In the present study, we extensively used differential UV/Visible spectroscopy as a useful tool to

75 demonstrate, qualitatively and quantitatively, whether interaction between two components

76 occurs or not. We found this technique very useful to probe the partition behavior of solubilizate

77 between solvent and micellar phase and to get the values of partition constant. In simple

$78 \mathrm{UV} /$ visible spectra, we made base line correction by keeping distilled water in both cells using

79 Hitachi, U-2800 spectrophotometer. Later on, the aqueous solution of dye was taken in sample

80 cell and its spectra were recorded at different dye concentration. Finally, the surfactant was

81 dissolved in dye solution and spectra were recorded at different surfactant concentration to

82 observe changes in dye spectra due to the presence of surfactants. For the differential

83 spectroscopy, initially, the aqueous solution of dye was prepared and divided it into two portions.

84 A part of this solution was used as reference and the other one was used to prepare surfactant

85 solution. The base line correction was made by keeping dye solution in the reference as well as 
86 sample compartments. Afterwards, a series of surfactant solutions were run to get differential

87 UV/Visible spectra of the respective compositions. ${ }^{[15,16,19]}$

88 Analytical grade, Cationic surfactant, Cetyltrimethyl ammonium bromide, CTAB and an anionic 89 surfactant Sodium Dodecyl Sulphate, SDS was purchased from Sigma Aldrich and were used as received, while Reactive dye, Reactive yellow 86 (IUPAC name, sodium 7-((4-(4,6-dichloro-

92 generously provided by Sandal dyestuff, Faisalabad, Pakistan. For all experiments, double

93 distilled water was used, which was processed using still apparatus (Model IM-100). The stock

94 solution of the dye was prepared in distilled water and was further diluted so that maximum

95 absorbance may not exceed 1 and the Lambert-Beer law could be applied. The concentration of 96 dye solution was kept at $1.5 \times 10^{-5} \mathrm{M}$ and surfactants were dissolved in it ranging from pre 97 micellar to post micellar concentration (from $0.8 \mathrm{mM}$ to $1.2 \mathrm{mM}$ for CTAB and from $7.5 \mathrm{mM}$ to $14.5 \mathrm{mM}$ for SDS).

99 The differential UV/Visible spectroscopy can be used to measure the absorbance of dye100 surfactant solution while removing the effect of dye absorbance at the same time. The increase in 101 value of differential absorbance $(\Delta A)$ with surfactant concentration suggests stronger dye102 surfactant interaction and indicates continuous inclusion of dye molecules within micelle. The 103 differential absorbance is zero below critical micelle concentration CMC and increases rapidly 104 beyond it. The values of $\Delta A$ at different surfactant concentration, above CMC, are inserted in 105 Kawamura equation to get value of partition coefficient that is measure of degree of 106 solubilization. ${ }^{[20-23]}$ 


\section{Partitioning of RY86 in CTAB micelles}

109 Figure 2(a) shows the simple UV/Visible spectrum of reactive yellow $86\left(1.5 \times 10^{-5} \mathrm{M}\right)$ in the 110 absence and presence of CTAB. The peak at $420 \mathrm{~nm}$ shows the maximum absorbance and was 111 selected for the subsequent study. The shift in spectra confirms the interaction between dye and 112 surfactant. The change in environment of dye molecules from polar water to non-polar core 113 causes red shift in the spectrum of dye containing solution. The cumulative effect of electrostatic 114 and hydrophobic interactions causes anionic dye molecules to be accommodated within the 115 micelles of cationic CTAB. From Figure 2(b), the observed increase in absorbance with 116 increasing concentration of CTAB indicates incorporation of large amounts of dye molecules 117 into the micelle of surfactant. The $C M C$ of CTAB is reduced (from $1.00 \mathrm{mM}$ to $0.89 \mathrm{mM}$ ) due to 118 two main factors (i) an increase in entropy upon mixing surfactant with solubilized dye in 119 micelle and (ii) decrease in electrical work of micellization due to decrease in repulsion between 120 surfactant heads caused by solubilized dye. The aforementioned factors enable dye to drive 121 surfactant unimers close to each other and initiate micellization before $C M C$. The $C M C$ of 122 CTAB in presence of RY86 was also determined by data of electrical conductivity, as displayed 123 in Figure 2(c). The value of $C M C$ obtained from UV/Visible spectroscopy $(0.89 \mathrm{mM})$ and 124 conductivity $(0.93 \mathrm{mM})$ are in close agreement with each other. The slight difference between 125 two $C M C$ values is due to the fact that $C M C$ does not have a sharp value rather it represents a 126 range of concentration within which micellization takes place. ${ }^{[19]}$ 

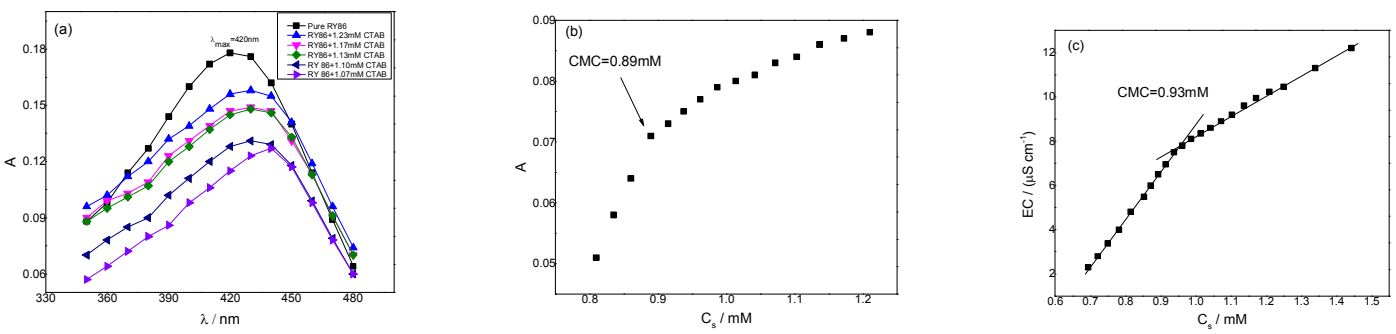

Figure 2. (a) Simple UV/Visible absorption spectra of Reactive yellow 86 in the presence of different $\mathrm{CTAB}$ concentration. (b) Plot of simple absorbance of Reactive yellow 86 as a function of CTAB concentration. (c) Plot of electrical conductivity of CTAB in presence of RY86 as a function of concentration at $298 \mathrm{~K}$.

The dye molecules are solubilized in outer portion of micelle and are under lateral pressure which tends to push it into the core of micelle. However, the attraction between anionic dye and cationic surfactant pushes the dye molecule in palisade layer very close to surface which thereby exhibits large $K_{x}$ value. This phenomenon of solvatochromism is indicative of penetration of dye into palisade layer of micelle. The attraction between opposite charges, however, does not allow deep penetration of dye into micelle. ${ }^{[20]}$ The initial decrease in absorbance could be due to surfactant-assisted self-aggregation of dye molecules that reduces repulsion between dye molecules. The subsequent increase in the absorbance could be due to adsorption of dye

141 molecules on the surface of oppositely charged CTAB. ${ }^{[21,23]}$ The results from absorbance study

142 reveal that the absorbance increases rapidly till $C M C$, while in post micellar region this increase 143 was slow due to maximum incorporation of dye molecule as indicated by the saturation plateau 144 in Figure 2(b) and 4(b). The slow increase in absorbance after $C M C$ can be explained on the 145 basis that greater amount of surfactant added in solution produces large number of micelles and 146 increase in absorbance is due to incorporation of dye molecules into newly formed micelles. ${ }^{[17,25,}$ $147 \quad 26-32]$ 
148 Initially, the interaction between dye anions $\mathrm{D}^{\mathrm{n}-}$ and surfactant cation $\mathrm{S}^{+}$produces dye-surfactant 149 ion pair complex, DS.

$$
\mathrm{D}^{\mathrm{n}-}+\mathrm{nS}^{+} \leftrightarrow \mathrm{DS}
$$

Self-aggregation of DS, causes absorbance to increase in premicellar region.

$$
\mathrm{n}(\mathrm{DS}) \leftrightarrow(\mathrm{DS})_{\mathrm{n}}
$$

At $C M C$, dye molecules enter into micelles as normal monomers and absorbance reaches its 154 limiting value.

$$
(\mathrm{DS})_{\mathrm{n}}+\mathrm{M} \leftrightarrow \mathrm{DM}
$$

$156 \mathrm{M}$ and DM represent empty micelles and dye containing micelle respectively.

157 Solubilization, being a dynamic process causes dye molecules to spend different residence time 158 at different positions between core and the surface because there is imbalance between the 159 hydrophilic and hydrophobic forces which do not let dye molecules to acquire a stable position 160 within micelle, rather keeps it fluctuating between surface and core, thus, giving random values 161 of absorbance. ${ }^{[13,14,17-18,21-24]}$

162 The position of dye molecules within micelle depends on the balance between hydrophilic and 163 hydrophobic forces. For anionic RY 86, hydrophilic forces are dominant and dye molecules are 164 oriented near the surface of micelle as evident from high value of partition coefficient for dye165 CTAB system. ${ }^{[29,30]}$

166 The long range electrostatic attraction between dye and surfactant molecules brings them close to 167 each other while the short range dispersive and the hydrophobic interactions are responsible to 168 align hydrophobic parts of dye and surfactant with each other in parallel fashion. The 169 aforementioned forces act cooperatively with each other. 
170 The qualitative as well as quantitative view of dye-surfactant interaction is provided by

171 differential spectroscopy. It helps to calculate the value of partition coefficient and free energy of

172 partition. The continuous increase in the differential absorbance with CTAB concentration

173 (Figure 3(a)) is indicative of continuous inclusion of additive molecules within micelle. ${ }^{[17-18,28,}$ $174 \quad 30]$

175 The value of partition coefficient was determined by the differential absorbance method reported 176 by Kawamura et al. ${ }^{[20]}$ according to the following equation;

$$
\frac{1}{\Delta A}=\frac{1}{K_{c} \Delta A_{\infty}\left(C_{d}+C_{s}^{m o}\right)}+\frac{1}{\Delta A_{\infty}}
$$

178 In equation $1, C_{d}$ is the concentration of dye in term of molarity; $C_{s}{ }^{m o}$ is analytical concentration 179 of surfactant calculated as $C_{s}-C M C_{o}$. Here, $C M C_{o}$ is $C M C$ of surfactant in water and $C_{s}$ is 180 surfactant concentration. $\Delta A$ is differential absorbance and $\Delta A_{\infty}$ represents its value at infinite 181 concentration of surfactant, where all dye molecules are supposed to have been solubilized. $K_{c}$ is 182 the partition constant having value in the units of $\mathrm{dm}^{3} / \mathrm{mol}$ calculated from slope of straight line 183 plotted according to equation 1 . The dimensionless quantity partition coefficient, $K_{x}$ is obtained 184 as $K_{x}=K_{c} n_{w}$, where $n_{w}$ is number of moles of water per $\mathrm{dm}^{3} \cdot{ }^{[17-18]}$ Figure 3(b) displays the plot 185 to calculate partition coefficient according to Kawamura model and table 1 shows data for 186 calculation of partition coefficient. 


\section{Table 1:}

188 Concentration of surfactant, $C_{s}$, differential absorbance, $\Delta A$, reciprocal of differential adsorption, $1891 / \Delta A$, analytical concentration of surfactant, $C_{s}^{m o}$ for calculation of partition coefficient of 190 reactive yellow 86 in CTAB micellar Media

\begin{tabular}{|c|c|c|c|c|c|}
\hline $\begin{array}{l}10^{3} C_{s} \\
(\mathrm{M})\end{array}$ & $10^{2} \Delta A$ & $1 / \Delta A$ & $\begin{array}{c}10^{5} C_{s}^{m o} / \\
(\mathrm{M})\end{array}$ & $10^{5}\left(C_{s}^{m o}+C_{d}\right) /$ & $\begin{array}{c}\left(C_{s}{ }^{m o}+C_{d}\right)^{-1} \\
(\mathrm{M})^{-1}\end{array}$ \\
\hline 1.21 & 2.7 & 37.03 & 20.96 & 22.47 & 4451.14 \\
\hline 1.17 & 2.5 & 40 & 17.18 & 18.68 & 5351.63 \\
\hline 1.14 & 2.3 & 43.48 & 13.64 & 15.13 & 6607.30 \\
\hline 1.10 & 2 & 50 & 10.29 & 11.79 & 8479.94 \\
\hline 1.07 & 1.6 & 62.5 & 7.14 & 8.64 & 11572.37 \\
\hline 1.05 & 1.4 & 71.43 & 4.16 & 5.66 & 17651.98 \\
\hline 1.01 & 1.1 & 90.91 & 1.35 & 2.85 & 35090.55 \\
\hline
\end{tabular}

192 The value of free energy change for the transfer of dye molecules from aqueous to micellar phase

193 was calculated using the following relation;

$$
\Delta \mathrm{G}_{\mathrm{p}}=-\mathrm{RT} \ln \mathrm{K}_{\mathrm{x}}
$$

195 In equation 2, " $R$ ” is the general gas constant and $T$ is absolute temperature. 
196 The large values of $K_{x}\left(1.12 \times 10^{6}\right)$ indicates large scale transfer of dye molecules from the 197 aqueous to the micellar media of CTAB, while, the large negative value of $\Delta G_{p}(-34.5 \mathrm{~kJ} / \mathrm{mol})$ is 198 an indicative of the spontaneous nature of partitioning and a sign of stability of system. ${ }^{[29,30]}$ 199 Scheme 1 indicates binding of RY86 molecules with CTAB while scheme 2(a) shows 200 partitioning of dye molecules with CTAB micelles.
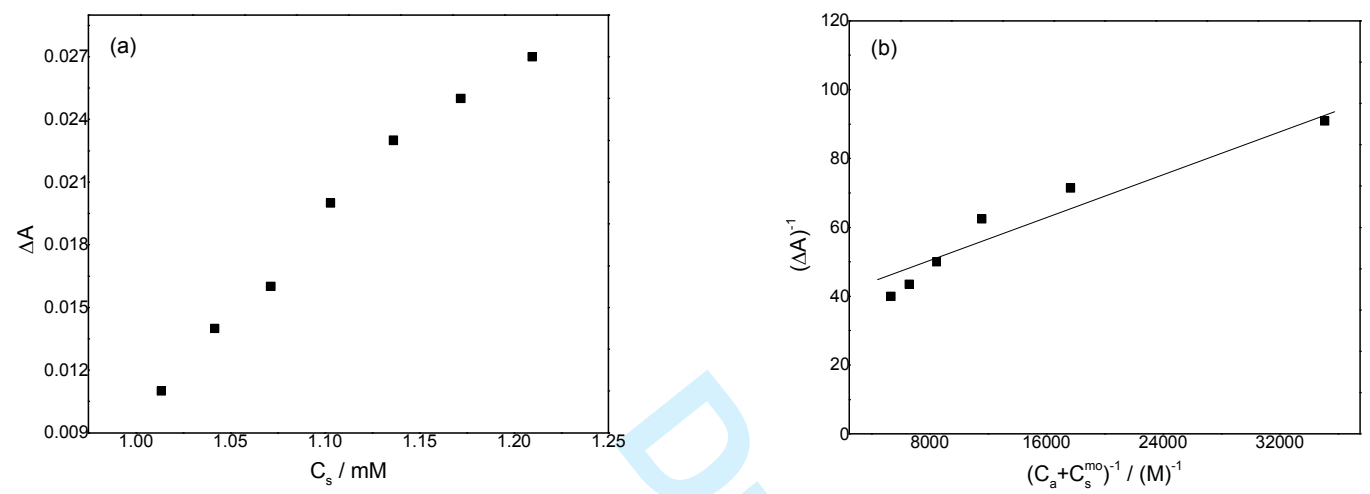

201

202

203

204

205

206

207

208

209

210

211

212

213

Figure 3. (a) Plot of differential absorbance of Reactive yellow 86 as a function of CTAB concentration. (b) Relationship between $(\Delta A)^{-1}$ and $\left(C_{d}+C_{s}^{m o}\right)^{-1}$ for the calculation of partition coefficient $\left(\mathrm{K}_{\mathrm{x}}\right)$ for Reactive yellow $86 / \mathrm{CTAB}$ system.

\section{Partitioning of RY86 in SDS micelles}

Figure 4(a) shows simple UV/Visible spectrum of reactive yellow $86\left(1.5 \times 10^{-5} \mathrm{M}\right)$ in the absence and presence of SDS. The shift in spectra indicates interaction between dye and surfactant; however, it is the repulsive interaction due to similar charges on dye and SDS. There is hypochromic shift but no bathochromic or hypsochromic shift because the polarity around head groups of dye remains same either it is in pure water or in SDS micelle. Similar charges on surface of SDS micelle and RY86 inhibit the dye to penetrate in micelle and keep dye at micelle surface $^{[14-18]}$ as indicated in scheme 2(b). Due to electrostatic repulsion between similar charges, 
214 the only force responsible for partitioning of dye in SDS micelle is hydrophobic interaction. The 215 increase in absorbance with concentration of SDS (Figure 4(b)) indicates limited partitioning of 216 dye molecules into the micelle as repulsion between similar charges opposes deep penetration. ${ }^{[23-}$ $217 \quad 24]$
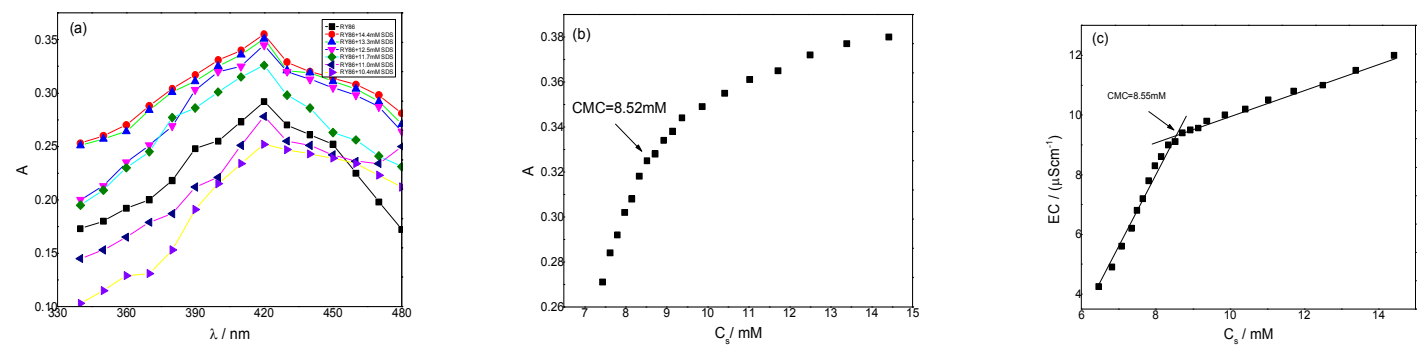

Figure 4. (a) Simple UV/Visible absorption spectra of Reactive yellow 86 in the presence of different SDS concentration. (b) Plot of simple absorbance of Reactive yellow 86 as a function of SDS concentration. (c) Plot of electrical conductivity of SDS in presence of RY86 as a function 222 of concentration at $298 \mathrm{~K}$.

Figure 5(a) shows continuous increase in the differential absorbance with increasing SDS concentration which is indicative of hydrophobic partitioning of dye molecules within micelle. ${ }^{[17-}$ 18, 28- 30]. Figure 4(b) and 4(c) shows that the dye increases CMC of SDS (from $8.2 \mathrm{mM}$ to $8.52 \mathrm{mM}$ ) which can be ascribed to the repulsion between similar charges on dye and SDS partially increasing electrical work of micellization. In fact, the presence of dye molecules 229 inhibits the close approach of surfactant molecules and causes delay in micellization. The 230 electrostatic repulsion compels dye to keep its anionic sulfonate groups out of micelle and dye 231 molecules remain half buried in micelle, as displayed scheme 2(b). When SDS was added to dye 232 solution no red or blue shift is observed because chromophores of dye are still in water 233 molecules and there is no change in polarity around chromophores. After reaching $C M C$, there is 
234 little increase in absorbance due to formation of more micelles and incorporation of dye 235 molecules in newly formed micelle causes little increase in absorbance. ${ }^{[17-18]}$ Similar models 236 regarding electrostatic and hydrophobic interaction by surfactants have been proposed by Ghosh 237 et al. ${ }^{[3,7]}$, Malik et al. ${ }^{[4]}$, Saha et al. ${ }^{[5]}$ and Sar, et al. ${ }^{[6]}$.

238 Figure 5(b) displays the plot to calculate partition coefficient according to Kawamura model and 239 table 2 shows data for calculation of partition coefficient.

240 Table 2:

241 Concentration of surfactant, $C_{s}$, differential absorbance, $\Delta A$, reciprocal of differential adsorption, $2421 / \Delta \mathrm{A}$, analytical concentration of surfactant, $C_{s}{ }^{m o}$ for calculation of partition coefficient of 243 reactive yellow 86 in SDS micellar Media
$10^{3} C_{s}$
$10^{5} C_{s}^{m o}$
$10^{5}\left(C_{s}^{m o}+C_{d}\right)$
$\left(C_{s}^{m o}+C_{d}\right)^{-1} /$

$10^{2} \Delta A \quad 1 / \Delta A$

(M)

(M)

(M)

$(\mathrm{M})^{-1}$

\begin{tabular}{lccccc}
\hline 13.4 & 2.8 & 35.7 & 519.28 & 520.78 & 192.018 \\
11.72 & 2.6 & 38.47 & 351.87 & 353.37 & 283 \\
11.03 & 2.4 & 41.67 & 282.94 & 284.43 & 351.57 \\
9.87 & 1.8 & 55.56 & 166.84 & 168.34 & 594.03 \\
9.15 & 1.3 & 76.93 & 94.63 & 96.13 & 1040.23 \\
8.72 & 0.9 & 111.11 & 52.09 & 53.59 & 1865.97 \\
\hline
\end{tabular}



8.52
0.7
142.85
32.27
33.77
2961.11

245 Table 3 shows the values of partition coefficient and free energy of partition for dye/SDS system.

246 The values of $K_{x}$ for SDS micelle is lower $\left(4.5 \times 10^{4}\right)$ than in CTAB micelles $\left(1.12 \times 10^{6}\right.$. This

247 indicates that the dye is partitioned in SDS micelle to lesser extent than in CTAB ones. The

248 negative value of $\Delta G_{p}(-26.38 \mathrm{~kJ} / \mathrm{mol})$ is an indicative of the spontaneous nature of partitioning

249 and a sign of stability of system. ${ }^{[29,30]}$
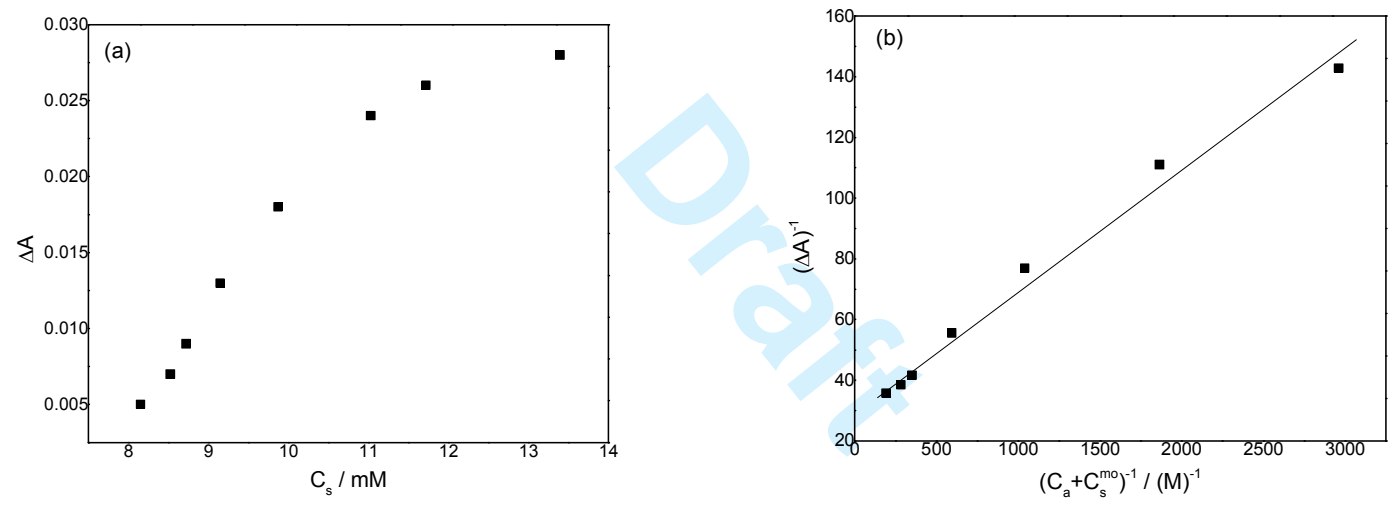

250

251 Figure 5. (a) Plot of differential absorbance of Reactive yellow 86 as a function of SDS 252 concentration. (b) Relationship between $(\Delta \mathrm{A})^{-1}$ and $\left(\mathrm{C}_{\mathrm{d}}+\mathrm{C}_{\mathrm{s}}^{\mathrm{mo}}\right)^{-1}$ for the calculation of partition 253 coefficient $\left(\mathrm{K}_{\mathrm{x}}\right)$ for Reactive yellow 86/SDS system. 


\section{Comparison of partitioning of RY86 within SDS and CTAB micelle}

257 RY86 is partitioned between aqueous and micellar media of CTAB to greater extent than in SDS

258 micelles. As shown in Scheme 1, the interaction between CTAB micelle and RY86 takes place 259 due to attraction between opposite charges. Dye molecules will initially adsorb at the surface of 260 micelle due to electrostatic interaction. However, this macromolecule has two aromatic rings 261 which have affinity for nonpolar core of micelle and therefore the electrostatic attraction alone is 262 not able to support it at surface. In fact, the dye molecules move into micelle in such a way that 263 their hydrophilic parts viz. sulfonate groups $\left(\mathrm{SO}_{3}{ }^{-}\right)$remain close to surface and aromatic rings 264 penetrate into core as evident from scheme 2(a). Dye molecules are, thus, solubilized into the 265 palisade layer of micelle.

267 Unlike incorporation of RY86 in CTAB micelles where both electrostatic as well as hydrophobic 268 forces are involved, partitioning of dye molecules within SDS micelles are favored by 269 hydrophobic forces only. In this scenario, the solubilization takes place to less extent than in case 270 of CTAB as clear from lower value of partition constant in SDS micelle $\left(4.5 \times 10^{4}\right)$ than in CTAB 271 micelle $\left(1.12 \times 10^{6}\right)$. Opposite charges on dye and CTAB causes larger negative value of $\Delta G_{p}$ in 272 case of dye/CTAB system, facilitating spontaneity of solubilization. While in case of dye/SDS 273 system, the repulsion between similar charges makes solubilization less spontaneous. If it 274 requires to remove dyes from water by micellar enhanced ultrafiltration, it is suggested to select 275 oppositely charged surfactant so that removal of impurity may be reinforced by electrostatic as 276 well hydrophobic forces. Dye spectra undergo red shift in presence of CTAB due to change in 
277 polarity around its chromophores while in SDS causes no red shift as there is no such change in 278 polarity.

279 Repulsive interaction by SDS head groups have also been proposed by Saha et al. ${ }^{[31]}$, Ghosh et 280 al. ${ }^{[32-34]}$ and Mukherjee et al. ${ }^{[35]}$.

281 
283 Table 3.

284 Partition coefficient, $K_{x}$ and free energy of partition, $\Delta G_{p}$; for reactive yellow $86 / \mathrm{CTAB}$ and 285 reactive yellow $86 /$ SDS systems.

\begin{tabular}{ccc}
\hline & & $\Delta G_{p} /$ \\
Systems & $K_{x}$ & $\left(\mathrm{~kJ} \mathrm{~mol}^{-1}\right)$ \\
\hline RY86-CTAB & $1.12 \times 10^{6}$ & -34.50 \\
RY86-SDS & $4.5 \times 10^{4}$ & -26.38 \\
\hline
\end{tabular}

286

287

288

289<smiles>CCN1C(=O)C(=NN)C(C)C(C(N)=O)C1=O</smiles><smiles>CNc1nc(Cl)nc(Cl)n1</smiles><smiles>CCCc1cc([S+](=O)[O-])c(S(=O)(=O)O)cc1[S+](=O)[O-]</smiles>

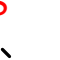

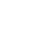




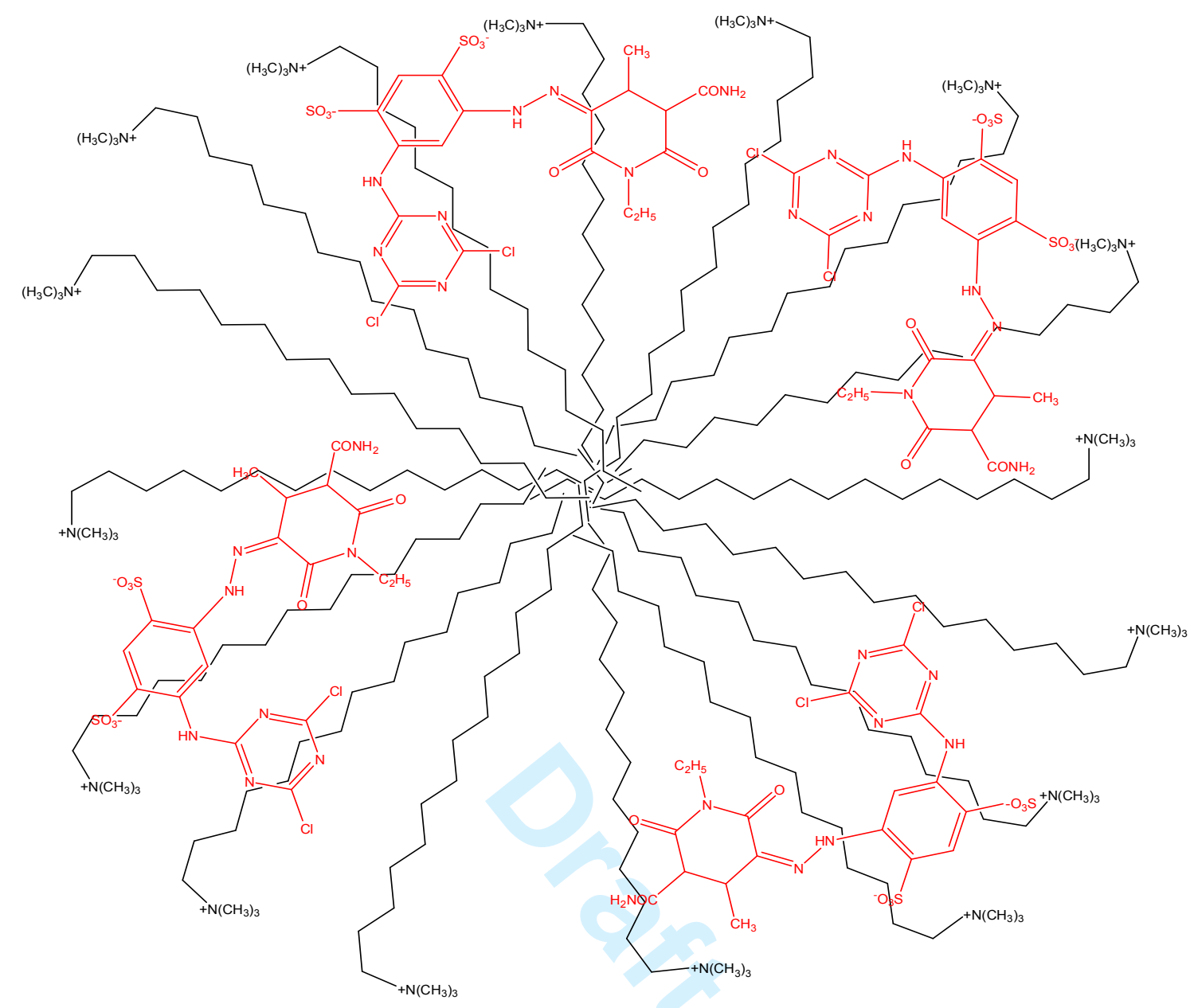

293 Scheme 2 (a). Partitioning of RY 86 in micelle of CTAB. 


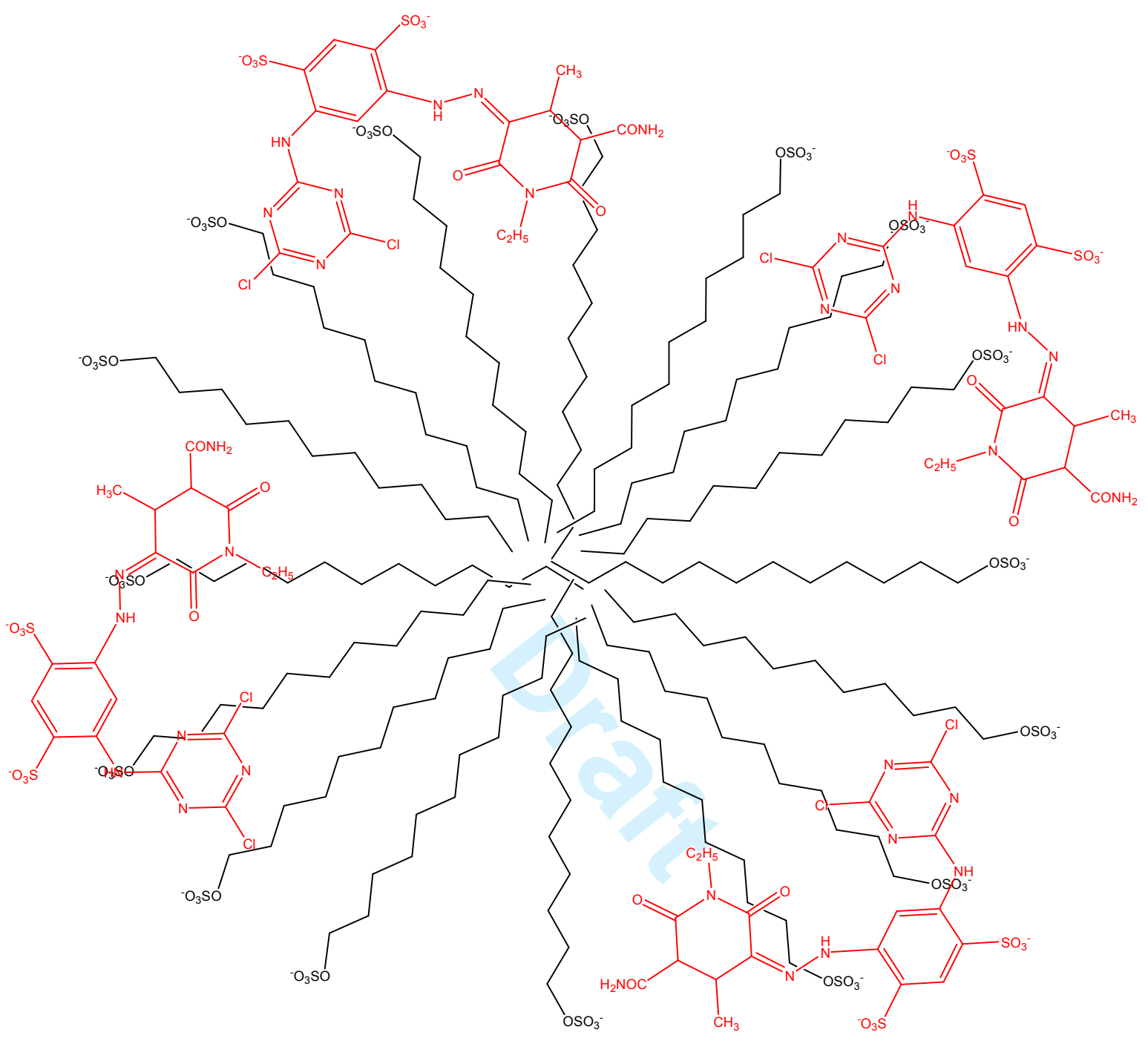

296

297 Scheme 2 (b). Partitioning of RY 86 in micelle of SDS

299 In the present study, the solubilization of anionic dye, reactive yellow 86 has been investigated in 300 the micellar media of CTAB (a cationic surfactant) and SDS ((an anionic surfactant). For this 301 purpose, simple and differential UV/Visible absorption spectra were recorded. The results 
302 revealed the higher degree of solubilization of reactive yellow 86 in micelles of cationic 303 surfactant (CTAB) than anionic SDS. The shift in UV/visible spectra of dye in the presence of $304 \mathrm{CTAB}$ is an indicative of strong interaction between the two phases. The dye has relatively 305 higher value of $K_{x}$ and more negative value of $\Delta G_{p}$ in CTAB micelles than in SDS micelles. It is 306 because both electrostatic and hydrophobic forces favor partitioning of dye in CTAB micelle 307 while solubilization in SDS micelle is governed by hydrophobic force only because electrostatic 308 repulsion hinders partitioning in SDS micelle.

\section{Acknowledgement}

310 This paper is a part of M.Phil thesis of Mr. Naveed ul Haq. All authors equally performed at

311 various stages of planning, execution and write up. U. A. Rana would like to extend his sincere

312 appreciation to the Deanship of Scientific Research at the King Saud University for its funding

313 of this research through the Prolific Research Group, Project No. PRG-1436-18. 


\section{References}

1. Lakra, J.; Tikariha, D.; Yadav, T.; Das, S.; Ghosh, S.; Satnami, M.L.; Ghosh, K.K.; Coll. Surf. A. 2014, 451, 56.U

2. Hosseinzadeh, R.; Maleki, R.; Matin, A.A.; Nikkhahi, Y.; Spectrochimica Acta Part A. 2008, 69,1183 .

3. Ghosh, A.; Sar, P.; Malik, S.; Saha, B.; J. Mol. Liq.. 2015, 211, 48.

4. Saha, R.; Ghosh, A.; Saha, B.; Chem. Eng. Sci. 2013, 99, 23.

5. Sar, P.; Ghosh, A.; Malik, S.; Ray, D.; Das, B.; Saha, B.; J. Ind. Eng. Chem. DOI: 10.1016/j.jiec.2016.07.028.

6. Malik, S.; Mondal, M.H.; Ghosh, A.; De, S.; Mahali, K.; Bhattacharyya, S.S.; Saha, B.; J. Solution Chem. 2016, 45, 1043.

7. Ghosh, A.; Saha, R.; Saha, B.; J. Ind. Eng. Chem. 2014, 20, 345-355.

8. Gul, F.; Khan, A.M.; Shah, S.S.; Nazar, M.F.; Color. Technol. 2010, 126, 109.

9. Dezhampanah, H.; Firouzi, R.; Int. J. Res. Phy. Chem. 2012, 2, 45.

10. Bagha, A.R.T.; Holmberg, K.; Materials 2013, 6, 580.

11. Matsuokaa, K.; Yamashitab, R.; Ichinoseb, M.; Kondob, M.; Yoshimurac, T.; Coll. Surf. A. 2014, 456, 83.

12. Takeuchia, E.; Matsuokab, M.; Ishiia, S.; Ishikawaa, C.; Hondaa, K.; Endoa, K.; Coll. Surf. A. 2014, 441, 133.

13. Naseem, B.; Sabri, A.; Hasan, A.; Shah, S.S.; Coll. Surf. A 2004, 35, 7.

14. Shah, S.S.; Laghari, S.S.; Naeem, K.; Thin solid films 1999, 346, 145.

15. Barbro, N.; Barni, E.; Barolo, E.; Quagliotto, P.; Viscardi, G.; Napione, L.; Pavan, S.; Bussolino, F.; Dyes. Pigm. 2008, 80, 307. 
16. Oakes, J.; Batchelor, S.N.; Dixon, S.; Color. Technol., 2005, 121, 237

17. Usman, M.; Siddiq, M.; J. Chem. Therm. 2013, 58, 359.

18. Usman, M.; Siddiq, M.; Spectrochimica Acta Part A 2013, 113, 182.

19. Shah, A.; Khan, A.M.; Usman, M.; Qureshi, R.; Siddiq, M.; Shah, S.S.; J. Chil. Chem. Soc. 2009, 54, 134.

20. Kawamura, H.; Manabe, M.; Miyamoto, Y.; Fujita, Y.; Tokunaga, S.; J. Phys. Chem. 1989, 93, 5536.

21. Shah, S.S.; Ahmad, R.; Shah, S.W.H.; Asif, K.M.; Naeem, K.; Coll. Surf A 1998, 137, 301.

22. Nazar, M. F.; Khan, A.M.; Shah, S.S.; J. Disp. Sci. Tech., 2010,31,596.

23. Nazar, M. F.; Shah, S.S.; Khosa, M.A.; J. Surf. Deterg., 2010, 13, 529.

24. Shick, M. J.; Fowkes, F. M.; J. Phys. Chem. 1957, 61, 1062.

25. Sarkar, M.; Poddar, S.; J. Coll. Interfac. Sci. 2000, 221, 181.

26. Khan, A.M.; Shah, S.S.; J. Disp. Sci. Technol. 2008, 29, 1401.

27. Akbas, H.; Taner, T.; Spectrochimica acta A., 2009, 73, 150.

28. Furias, T.; Menorval, L.C.; Zajac, J.; Rivera, A.; Coll. Surf. A 2009, 345, 51.

29. Shah, S.S; Laghari, G.M.; Naeem, K.; Shah, S.W.H.; Coll. Surf. A 1998, 143, 111.

30. Shah, S.S; Laghari, G.M.; Naeem, K.; Shah, S.W.H.; Coll. Surf. A 2000, 168, 77.

31. Saha, R.; Ghosh, A.; Sar, P.; Saha, I.; Ghosh, S.K.; Mukherjee, K.; Saha , B.;Spectrochimica Acta Part A: 2013, 116, 524.

32. Ghosh, A.; Sengupta, K.; Saha, R.; Saha , B.,J. Mol. Liq, 2014, 198, 369.

33. Ghosh, A.; Saha, R.; Mukherjee, K.; Sar, P.; Ghosh, S.K.; Malik, S.; Bhattacharyya, S.S.; Saha , B.; J. Mol. Liq, 2014,190, 81. 
34. Ghosh, A.; Saha, R.; Sar, P.; Saha, B.,J. Mol. Liq, 2013, 186, 122.

363

35. Mukherjee, K.; Saha, R.; Ghosh, A.; Ghosh, S.K.; Saha, B.; J. Mol. Liq.2013,

364 179, 1 
https://mc06.manuscriptcentral.com/cjc-pubs 